\title{
GGMncv: Nonconvex Penalized Gaussian Graphical Models in R
}

\author{
Donald R. Williams
}

\begin{abstract}
Studying complex relations in multivariate datasets is a common task across the sciences. Recently, the Gaussian graphical model has emerged as an increasingly popular model for characterizing the conditional dependence structure of random variables. Although the graphical lasso ( $\ell_{1}$-regularization) is the most well-known estimator, it has several drawbacks that make it less than ideal for model selection. There are now alternative forms of regularization that were developed specifically to overcome issues inherent to the $\ell_{1}$-penalty. To date, however, these alternatives have been slow to work their way into software for research workers. To address this dearth of software, I developed the package GGMncv that includes a variety of nonconvex penalties, two algorithms for their estimation, plotting capabilities, and an approach for making statistical inference. As an added bonus, GGMncv can be used for nonconvex penalized least squares. After describing the various nonconvex penalties, the functionality of GGMncv is demonstrated through examples using a dataset from personality psychology.
\end{abstract}

\section{Introduction}

Studying complex relations in multivariate datasets is a common task across the sciences. Cognitive neuroscientists model brain connectivity with the goal of unearthing functional and structural associations between cortical regions (Ortiz et al., 2015). In clinical psychology, researchers study the intricate web of symptom interrelations that underlie mental health disorders (McNally, 2016; Borsboom et al., 2011). Econometricians seek to understand the international "financial flow" among countries (Giudici and Spelta, 2016). To this end, Gaussian graphical model (GGM) has emerged as an oft-used tool, where the basic idea is to characterize multivariate relations by learning the conditional dependence structure

Inherent to Gaussian graphical modeling is the problem of covariance selection (Dempster, 1972), in that the off-diagonal elements of the inverse covariance (precision) matrix capture the dependence structure, that, when standardized and signed reversed, yield partial correlation coefficients. Even with a moderate number of variables, this can pose computational challenges, because, with $p$ variables, there are $\frac{1}{2} \cdot p(p-1)$ potential relations. ${ }^{1}$ This translates into $2^{\frac{1}{2}} p(p-1)$ candidate graphs (Mohammadi and Wit, 2015). As a result, algorithms are continuously developed with the goals of accurate model selection and computational feasibility (for but a few of the possibilities see Fan et al., 2016).

Although the most popular estimator is the graphical lasso (a.k.a., "least absolute shrinkage and selection operator," Tibshirani, 1996; Friedman et al., 2008; Witten et al., 2011), it has long been known that the $\ell$-penalty has several drawbacks, including that it is less than ideal for model selection. In fact, Mazumder et al. (2011) explicitly noted the following:

...greedier methods like subset regression and the nonconvex methods we discuss here achieve sparser models than the lasso for the same or better prediction accuracy, and enjoy superior variable-selection properties (p. 1125).

A drawback of subset selection, that is, the $\ell_{0}$ (puesdo) norm, is computational in nature, in that it entails an intensive combinatorial search. There are now a variety of nonconvex penalties, including those that approximate the $\ell_{0}$-penalty, that (mostly) retain the computational attractiveness of $\ell_{1}$-regularization. In addition, superior performance for model selection in GGMs was recently demonstrated in Williams (2020)

To date, however, these insights have been slow to work their way into software. For example, the vast majority of software that implements nonconvex penalties is for regression modeling, including the MCP and SCAD in the popular R package ncvreg (Breheny and Huang, 2011). Furthermore, the recent $R$ package ncpen implements a total seven nonconvex penalties for regression (Kim et al., 2018). To my knowledge, there are no R packages that specifically feature nonconvex penalties for GGMs. A honorable mention is pgraph that includes the SCAD, but note that the package was built for a newly developed projection-based method (Fan et al., 2020). To address this dearth of software, I developed the R package GGMncv that includes a variety of nonconvex penalties and two algorithms for their estimation.

\footnotetext{
${ }^{1}$ In the ubiquitous case of model selection in regression there are only $p$ potential coefficients (the number of possible models is $2^{p}$ ).
} 


\section{Model Formulation}

For multivariate normal data (Baba et al., 2004; Baba and Sibuya, 2005), a GGM captures conditional relations that are typically visualized to infer the underlying dependence structure (i.e., the partial correlation "network"; Højsgaard et al., 2012; Lauritzen, 1996). There is an undirected graph that is denoted $G=(V, E)$, consists of a vertex $V=\{1, \ldots, p\}$ and an edge set $E \subset V \times V$. The former refers to "nodes" that are, say, brain regions, whereas $E$ is the estimated dependence structure. Let $\mathbf{y}=\left(y_{1}, \ldots, y_{p}\right)^{\prime}$ be a random vector indexed by the graph's vertices that is assumed to follow a multivariate normal distribution, $\mathbf{y} \sim \mathcal{N}_{p}(\boldsymbol{\mu}, \boldsymbol{\Sigma})$, with the mean vector $\boldsymbol{\mu}=\left(0_{1}, \ldots, 0_{p}\right)^{\prime}$ and a $p \times p$ positive definite covariance matrix $\Sigma$.

The undirected graph is obtained by determining which off-diagonal elements in the precision matrix, $\boldsymbol{\Theta}=\boldsymbol{\Sigma}^{-1}$, are nonzero. That is, $(i, j) \in E$ when nodes $i$ and $j$ are determined to be conditionally dependent and set to zero otherwise. Note that standardizing $\Theta$ and reversing the sign yields partial correlations, that is,

$$
\rho_{i j \cdot z}=\frac{-\theta_{i j}}{\sqrt{\theta_{i i} \theta_{j j}}},
$$

where $z$ contains the nodes conditioned on (i.e., $p-2$ ). Note that it is possible to determine $E$ by using Bayesian (Williams and Mulder, 2020) or frequentist hypothesis testing for each partial correlation (Drton and Perlman, 2005). When using nonconvex and $\ell_{1}$ - regularization (that is convex), however, the standard approach is to work with the inverse correlation matrix, $\mathbf{R}^{-1}$, that is,

$$
\boldsymbol{\Theta}=\mathbf{R}^{-1}=(d \Sigma d)^{-1},
$$

where $\boldsymbol{d}$ is a diagonal matrix with $d_{i i}=1 / \sqrt{\sigma_{i i}}$. This is done to ensure that the parameters are on the same scale. In what follows, the off-diagonal elements of $\boldsymbol{\Theta}$ are denoted as $\theta$.

\section{Nonconvex Penalized Likelihood}

To estimate the conditional dependence structure for a broad class of nonconvex penalties, ${ }^{2}$ the penalized likelihood is defined as

$$
l\left(\mathbf{R}^{-1}\right)=\log \operatorname{det} \mathbf{R}^{-1}-\operatorname{tr}\left(\mathbf{R} \mathbf{R}^{-1}\right)-\sum_{i \neq j} p_{\lambda_{i j, \gamma}}\left(\left|\mathbf{R}_{i, j}^{-1}\right|\right),
$$

where $\mathbf{R}$ is the sample correlation matrix and $p_{\lambda, \gamma}($.$) is a generic penalty function (Fan et al., 2009), with$ the regularization parameter, $\lambda$, that controls the amount of penalization, such that there are a range of possibilities, depending on $\lambda$ and $\gamma$. The additional parameter $\gamma$ influences the shape of the penalty (Breheny and Huang, 2011). One point of emphasis is the subscripts on $\lambda_{i j}$ that effectively allows each parameter to be differentially regularized. It is this feature that differentiates nonconvex from $\ell_{1}$-regularization. Indeed, by replacing $p_{\lambda, \gamma}($.$) with p_{\lambda}($.$) in Equation 3$, this results in the $\ell_{1}$-penalized likelihood.

\section{Nonconvex Penalties}

In the verbiage of Fan and Li (2001), the following penalties enjoy the so-called oracle properties:

1. The true model is selected, with a probability tending to 1 , as the sample size becomes large.

2. The parameters are asymptotically normal and they have the same asymptotic variance as non-regularized estimation.

In other words, "the penalized likelihood estimators work as well as if the correct submodel were known in advance" (p. 1348, Fan and Li, 2001).

\section{Customary Nonconvex Penalties}

Smoothly Clipped Absolute Deviation: In Fan and Li (2001), it was conjectured that $\ell_{1}$-regularization does not enjoy the oracle property. Their theoretical results demonstrated that the $\ell_{1}$-penalty can be

\footnotetext{
${ }^{2}$ This general class includes those nonconvex penalties with two tuning parameters.
} 
consistent for model selection and consistently estimate the parameters, but it cannot satisfy both properties simultaneously (see Theorem 2 and Remark 1 in Fan and Li, 2001).

To achieve both properties simultaneously, Fan and Li (2001) proposed the SCAD penalty defined on $[0, \infty]$, that is,

$$
p_{\lambda, \gamma}(\theta) \begin{cases}\lambda|\theta| & \text { if }|\theta| \leq \lambda \\ \frac{2 \gamma \lambda|\theta|-\theta^{2}-\lambda^{2}}{2(\gamma-1)} & \text { if } \lambda<|\theta| \leq \gamma \lambda \\ \frac{\lambda^{2}(\gamma+1)}{2} & \text { if }|\theta|>\lambda \gamma\end{cases}
$$

for $\gamma>2$. Notice that the penalty is clearly a function of $\theta$ (Figure 1 ). On the other hand, the $\ell_{1}$-penalty function is simply $\lambda|\theta|$. Although the formulation in Equation (4) opens the door for various possible combinations of $\lambda$ and $\gamma$, setting $\gamma=3.7$ is a common choice that was motivated by minimizing Bayesian risk (p. 1351, Fan and Li, 2001).

In addition to the oracle properties, Fan and Li (2001) outlined three more desirable characteristics for a penalty, each of which is satisfied by the SCAD:

1. Unbiasedness: The resulting estimator is nearly unbiased when the true unknown parameter is large to avoid unnecessary modeling bias.

2. Sparsity: The resulting estimator...automatically sets small estimated coefficients to zero to reduce model complexity.

3. Continuity: The resulting estimator is continuous...to avoid instability in model prediction (p. 1349, Fan and Li, 2001).

These are important to acknowledge because they have served as benchmarks for developing nonconvex penalties over the years. Indeed, all of the following penalties have these three characteristics, in addition to the oracle properties.

Minimax Concave Penalty: Another popular nonconvex penalty is the MCP that was introduced in (Zhang, 2010). The penalty function, again defined on $[0, \infty]$, is written as

$$
p_{\lambda, \gamma}(\theta) \begin{cases}\lambda \theta-\frac{\theta^{2}}{2 \gamma} & \text { if } \theta \leq \gamma \lambda \\ \frac{1}{2} \gamma \lambda^{2} & \text { if } \theta>\gamma \lambda\end{cases}
$$

for $\gamma>1$. It is common to set $\gamma$ to 2.0, although selecting it is also a possibility. The MCP closely resembles the SCAD, that is, "Both penalties begin by applying the same rate of penalization as the lasso, and reduce that rate to 0 as $\theta$ gets further away from zero" (p. 237, Breheny and Huang, 2011). To make sense of this, it is informative to consider the derivative of Equation 5, that is,

$$
p_{\lambda, \gamma}^{\prime}(\theta) \begin{cases}\lambda-\frac{|\theta|}{\gamma} & \text { if } \theta \leq \gamma \lambda \\ 0 & \text { if }|\theta|>\gamma \lambda\end{cases}
$$

Hence, when $|\theta|$ is greater than $\lambda \gamma$, the rate of penalization is zero. Note one key distinction between the SCAD and MCP is when the penalty is relaxed for increasingly larger values. This is illustrated in Williams (2020, see Figure 1 therein).

\section{Approximating the $\ell_{0}$-Penalty}

A desirable penalty would closely mimic the behavior of best subset selection, which is often considered the "gold-standard" for model selection. Although this is not accomplished by either the SCAD or $\mathrm{MCP}$, there are a variety of nonconvex penalties that were designed specifically to approximate the $\ell_{0}$-penalty, that is,

$$
p_{\lambda}(\theta)=\lambda I\{\theta \neq 0\}= \begin{cases}\lambda & \text { if } \theta \neq 0 \\ 0 & \text { if } \theta=0 .\end{cases}
$$

This penalty is ideal because the "estimators are penalized according to the number of nonzero parameter estimates; thus, model complexity is penalized in a straightforward and intuitive way" ( $\mathrm{p}$. 929, Dicker et al., 2013). The major hurdels are computational, due to having to enumerate all possible models, and the solution is discontinuous rather than exhibiting continuity which can translate into 
the estimator being relatively unstable (p. 2354 in Breiman, 1996). There are now a variety of penalties that provide a computationally efficient and continuous approximation to Equation (7).

Seamless $\ell_{0}$ : Initially for multiple regression, Dicker et al. (2013) introduced the seamless $\ell_{0}$-penalty (SELO). This can be written as

$$
p_{\lambda, \gamma}(\theta)=\frac{\lambda}{\log (2)} \log \left(\frac{|\theta|}{|\theta|+\gamma}+1\right)
$$

for $\gamma>0$. Note that when $\gamma \rightarrow 0$, say, $\gamma=0.01$, this approximates $p_{\lambda}(\theta) \approx \lambda I\{\theta \neq 0\}$ in Equation (7). Hence, $\gamma$ provides the desired property of continuity that overcomes this issue of best subsets. It is common to set $\gamma=0.01$, which was shown to work well in GGMs (Williams, 2020).

ATAN: In Wang and Zhu (2016), an arctangent penalty was introduced (ATAN). The ATAN penalty function is defined on $[0, \infty)$ and it is written as

$$
p_{\lambda, \gamma}(|\theta|)=\lambda\left(\gamma+\frac{2}{\pi}\right) \arctan \left(\frac{|\theta|}{\gamma}\right),
$$

for $\gamma>0$. Wang and Zhu (2016) suggested fixing $\gamma$ to 0.01. A key feature of Equation (9) is that there is a direct relationship between the $\ell_{0}$ and $\ell_{1}$ penalties. That is, as $\gamma \rightarrow \infty$, this results in $\ell_{1}$-regularization. On the other hand, as $\gamma \rightarrow 0$, this approximates the $\ell_{0}$-penalty. This differentiates the ATAN from the SELO penalty, as $\gamma$ in the SELO does not govern a continuous transition from the $\ell_{1}$ to $\ell_{0}$ penalties. This is illustrated in Figure 1.

Exponential: Termed the exponential penalty (EXP), Wang et al. (2018) proposed the following penalty

$$
p_{\lambda, \gamma}(|\theta|)=\lambda\left(1-\exp ^{\frac{|\theta|}{\gamma}}\right)
$$

for $\gamma>0$. Note that when $\gamma \rightarrow 0$, say, $\gamma=0.01$, this also results in $p_{\lambda, \gamma}(\theta) \approx \lambda I\{\theta \neq 0\}$, that is, a continuous approximation to the $\ell_{0}$-penalty. Similar penalties can be found in VanDerwerken (p. 11, 2011) and Breheny (Equation 6 therein, 2015), with the latter for group lasso.

Smooth Integration of Counting and Absolute Deviation: The smooth integration of counting and absolute deviation penalty (SICA) was introduced in (Equation 9 in Lv et al., 2009). The basic idea was to modify the transformed $\ell_{1}$-penalty of Nikolova (2000), such that it provides a family of penalties. The SICA penalty, noting that $\theta \in(0, \infty)$, is defined as

$$
p_{\lambda, \gamma}(|\theta|)=\lambda\left(\frac{|\theta|(\gamma+1)}{|\theta|+\gamma}\right)
$$

for $\gamma \in[0, \infty)$. Figure 1 in Lv et al. (2009) visualizes the penalty function. The resulting family of penalties "has the interpretation of a smooth homotopy between $\ell_{0}$ and $\ell_{1}$ penalties" (p. $3502 \mathrm{in} \mathrm{LV}$ et al., 2009). Note that this is also the idea behind ATAN penalties.

\section{Illustrative Penalty Functions}

To make sense of the various penalties, it is informative to visualize their function with respect to $\theta$. To this end, GGMncv includes the function penalty_function() that computes a penalty function, which can then be plotted with plot(). Note all plots in GGMncv are made with ggplot2 (Wickham, 2016). As of version 2.0.0, ATAN is the default penalty. Its penalty function is computed and plotted as follows:

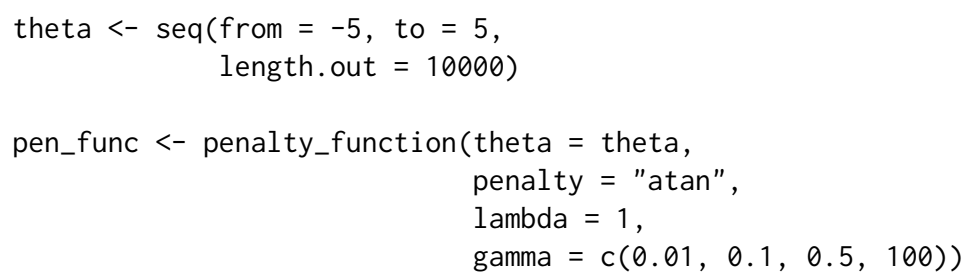



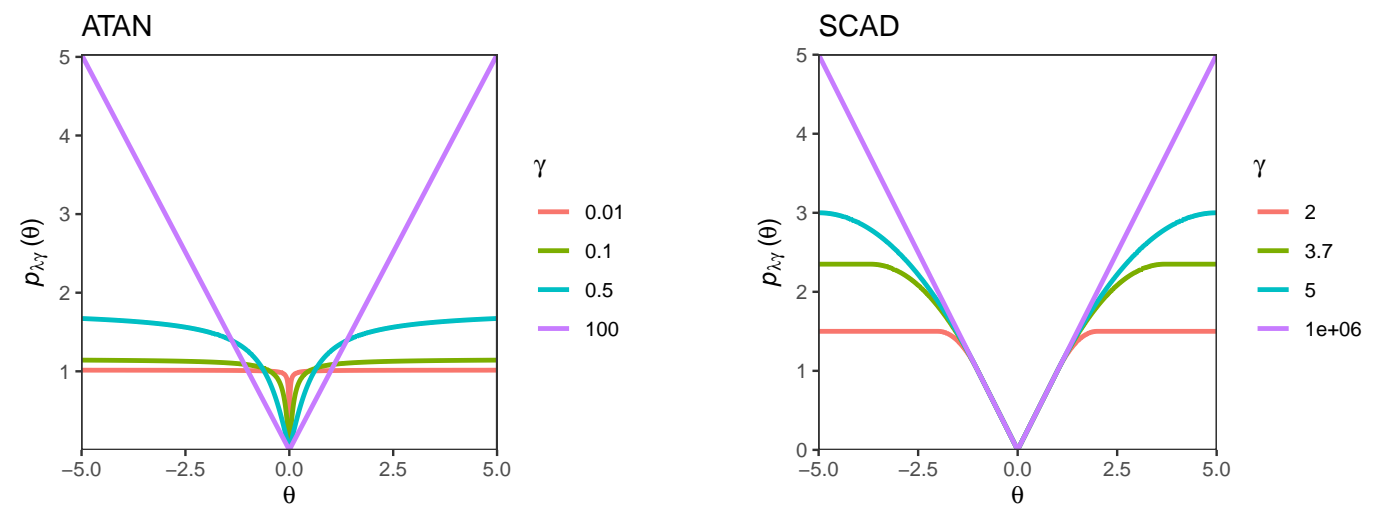

Figure 1: Illustrative penalty functions $p_{\lambda \gamma}(\theta)$.

pen_plot <- plot(pen_func)

The pen_plot object is of class ggplot which allows for further customization:

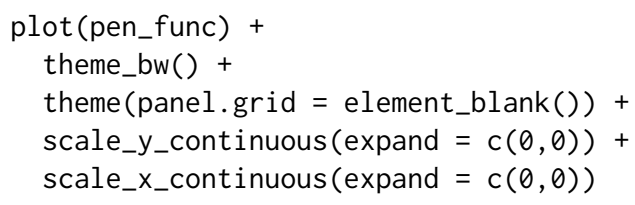

This is visualized in Figure 1. It is readily seen that, as $\gamma \rightarrow 0$, the function is a continuous approximation to the $\ell_{0}$-penalty, whereas, with a larger value (e.g., $\left.\gamma=100\right)$, it approximates the $\ell_{1}$-penalty. This intuitive interpretation is the reason it is currently the default, given the performance of these nonconvex penalties are comparable (Williams, 2020). As an additional example, Figure 1 also includes the SCAD which approaches the $\ell_{1}$-penalty as $\gamma \rightarrow \infty$. The derivative of the penalty, $p_{\lambda, \gamma}^{\prime}(\cdot)$, can be computed with penalty_derivative() and similarly visualized with plot().

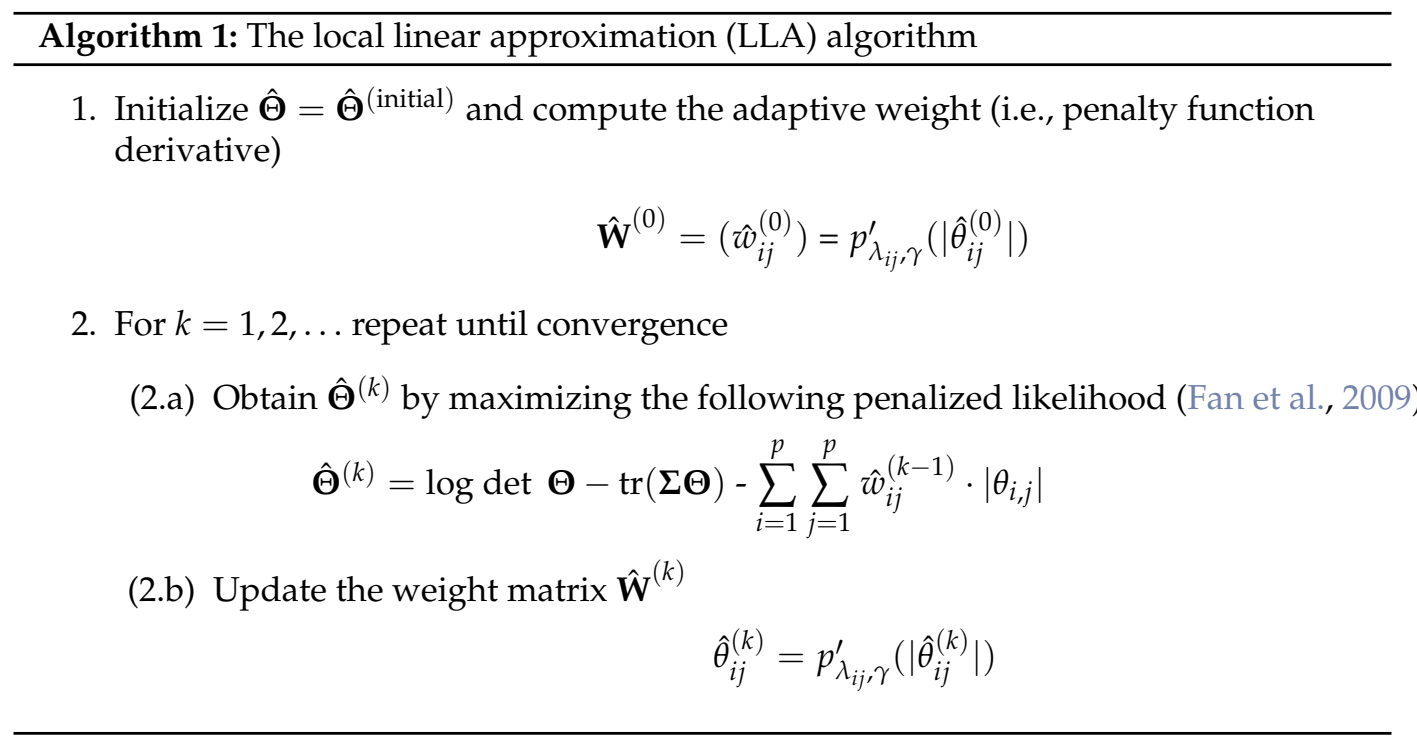

\section{Computational Details}

\section{Local Linear Approximation}

Zou and Li (2008) is a seminal work for computing the nonconvex penalized likelihood. The first advancement was an iterative algorithm based on local linear approximation (LLA). In GGMs, Fan et al. (2009) demonstrated that employing nonconvex penalties in GGMs can be recast in terms of 
iteratively weighted $\ell_{1}$-regularization, that is, at each step of the LLA, coordinate-descent algorithms (e.g., those implemented in glassoFast, Sustik et al., 2018) can accommodate nonconvex penalties. The LLA is provided in Algorithm 1.

\section{One-Step Estimator}

The second major contribution of Zou and Li (2008) was describing one-step estimators. A drawback of a fully iterative LLA (Algorithm 1) is that reaching convergence can require a large number of steps (iterations), each of which requires solving Equation 3 and computing the derivative of the penalty function. The one-step estimator requires computing each only once. This is accurate "provided that the initial estimates are good enough" (p. 1511, Zou and Li, 2008). In low-dimensional settings $(p<n)$, however, using the MLE is recommended for the initial estimate of $\Theta$. In high-dimensional settings, it is possible to specify initial (in the main function $\operatorname{ggmncv}($ )) to a custom starting value for $\Theta$ (e.g., a shrinkage estimator Ledoit and Wolf, 2004).

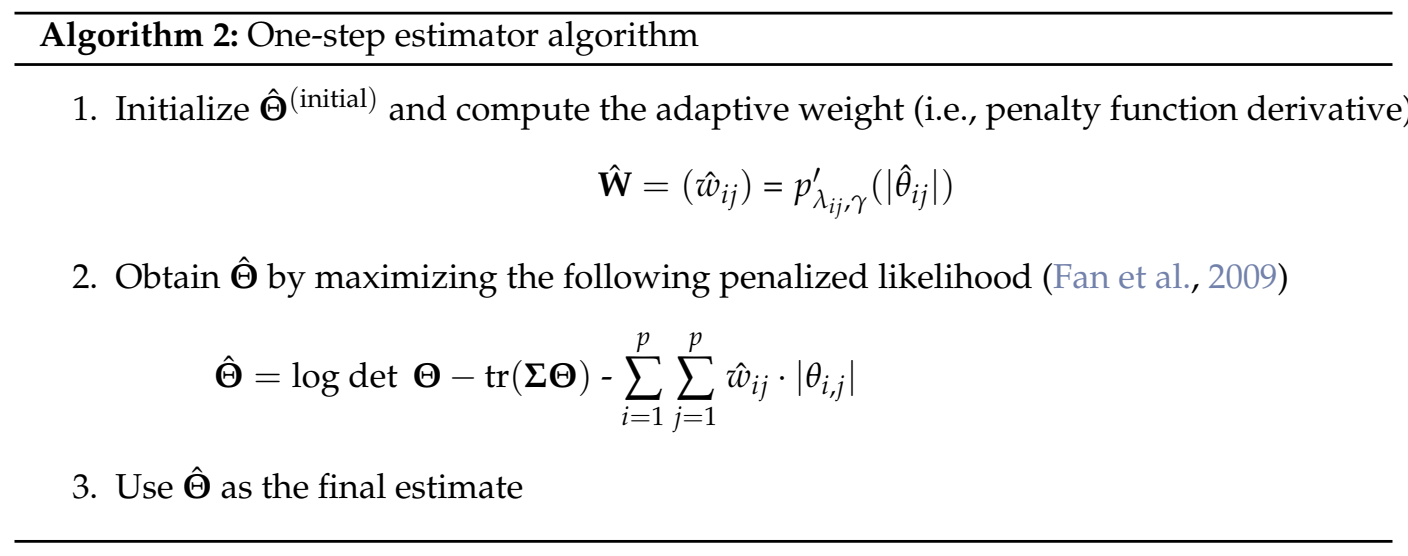

\section{Tuning Parameter Selection}

It is commonplace to select a model according to minimizing (or maximizing) some information criterion. In GGMncv, there are a variety of options for selecting $\lambda$ (select = "lambda"; the default), $\gamma($ select $=$ "gamma"), or both (select = "both"). Following Kim et al. (2012), it is thus informative to consider a generic formulation for negative 2 times the log-likelihood, that is,

$$
-2 l_{n}(\hat{\boldsymbol{\Theta}})=-2\left[\frac{n}{2} \log \operatorname{det} \hat{\boldsymbol{\Theta}}-\operatorname{tr}(\hat{\mathbf{S}} \hat{\boldsymbol{\Theta}})\right]
$$

where $\hat{\boldsymbol{\Theta}}$ is the estimated precision matrix (e.g., for a given $\lambda$ and $\gamma$ ) and $\hat{\mathbf{S}}$ is the sample-based covariance matrix. With Equation 12 in hand, it is straightforward to define a variety of GICs, with each as a penalty term added to Equation 12:

1. $\mathrm{GIC}_{1}:|\mathbf{E}| \cdot \log (n)$. Note that $|\mathbf{E}|$ denotes the number of edges (ic = "bic" or ic = "gic_1"). This is the Bayesian information criterion (Schwarz and others, 1978).

2. $\mathrm{GIC}_{2}:|\mathbf{E}| \cdot p^{1 / 3}$ (ic = "gic_2").

3. $\mathrm{GIC}_{3}:|\mathbf{E}| \cdot 2 \cdot \log (p)(\mathrm{ic}=$ "ric" or ic = "gic_3"). This is the Risk Inflation Criterion of Foster and George (1994).

4. $\mathrm{GIC}_{4}:|\mathbf{E}| \cdot 2 \cdot \log (p)+\log (\log (p))$ (ic = "gic_4").

5. $\mathrm{GIC}_{5}:|\mathbf{E}| \cdot \log (p)+\log (\log (n)) \cdot \log (p)($ ic $=$ "gic_5").

6. $\mathrm{GIC}_{6}:|\mathbf{E}| \cdot \log (n) \cdot \log (p)($ ic = "gic_6").

Additionally, GGMncv also includes the Akaike Information Criterion (ic = "aic", Akaike, 1974) and the extended BIC (ic = "ebic", Chen and Chen, 2008; Foygel and Drton, 2010). I refer interested readers to Kim et al. (2012) for a thorough discussion of the theory underlying GICs. The default in GGMncv is set to ic = "bic". For a sparser (denser) model, a GIC can be used with a larger (smaller) penalty term added to Equation 12. 


\section{Statistical Inference}

Although model selection is a customary practice in applied science, what is less appreciated (or not considered at all) is that "[after model selection] such inference enjoys none of the guarantees that classical statistical theory provides for tests and confidence intervals" (p. 802, Berk et al., 2013). This applies to both regularized and non-regularized estimation, in that corrections are needed to make post-selection inference. For an overview of this topic, I refer interested readers to Taylor and Tibshirani (2015).

\section{Debiased Estimator}

Avoiding model selection circumvents the issue of post-selection inference. The remaining issue for making statistical inference is overcoming the inherent bias and sparsity of the regularized estimator. This was first worked out for regression (Javanmard and Montanari, 2013, 2015), and, more recently, it was extended to glasso in Janková and van de Geer (2015). Note that this is sometimes referred to as depsarsiying (Van De Geer et al., 2014) The debiased glasso estimator, $\hat{\mathbf{T}}$, is defined as

$$
\hat{\mathbf{T}}=2 \hat{\boldsymbol{\Theta}}-\hat{\boldsymbol{\Theta}} \hat{\mathbf{R}} \hat{\boldsymbol{\Theta}}
$$

with $\hat{\boldsymbol{\Theta}}$ the glasso estimator of the precision matrix and $\hat{\mathbf{R}}$ the sample-based correlation matrix. As the name implies, this step removes the zeros and attempts to correct the bias from the $\ell_{1}$-penalty. The asymptotic variance is then given as

$$
\operatorname{Var}[\hat{\mathbf{T}}]=\operatorname{diag}(\hat{\mathbf{T}}) \operatorname{diag}(\hat{\mathbf{T}})^{\prime}+\hat{\mathbf{T}}^{2}
$$

where $\operatorname{diag}(\hat{\mathbf{T}})$ is a diagonal matrix with $\operatorname{diag}\left(t_{11}, \ldots, t_{p p}\right)$. This readily allows for computing $p$-values for each off-diagonal element of the desparsified estimator. As described below, this can be used to either determine $E$ with, say, false discovery control, or to confirm those edges detected with data-driven selection in an independent dataset.

\section{Illustrative Examples}

GGMs have recently emerged in personality research (e.g., Costantini et al., 2015). In what follows, I focus on the conditional dependence structure of 25 items that measure the big five inventory (Revelle, 2019)

\section{Data-Driven Model Selection:}

The standard way of estimating GGMs, that is, with data-driven model selection, is implemented as follows:

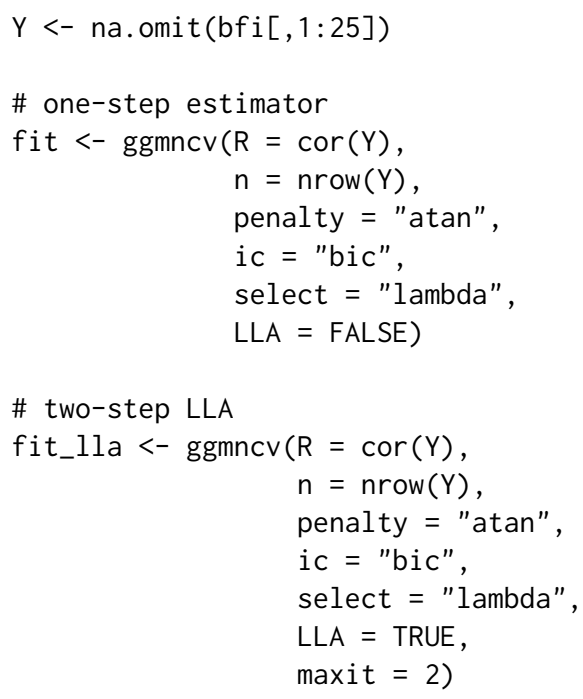

The object fit is the one-step estimator (Algorithm 2) and the object fit_lla is a two-step LLA (Algorithm 1). The argument maxit controls the number of steps in the LLA. The algorithm is 


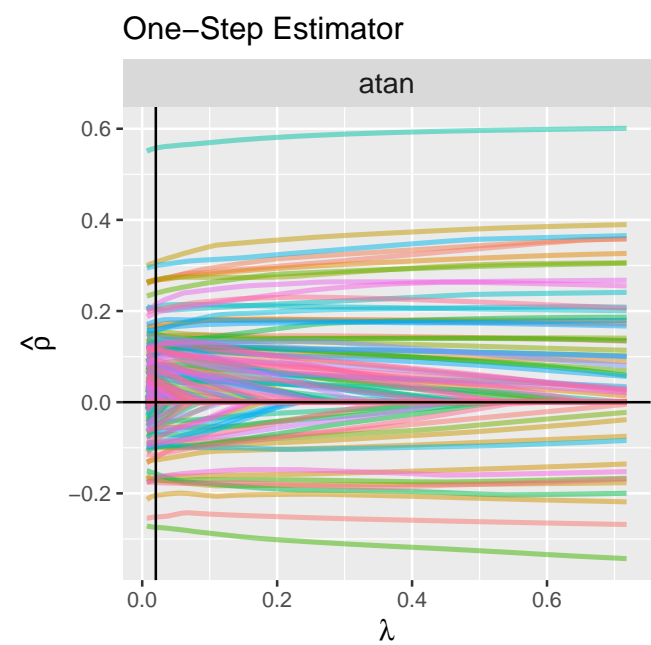

Conditional Dependence Structure

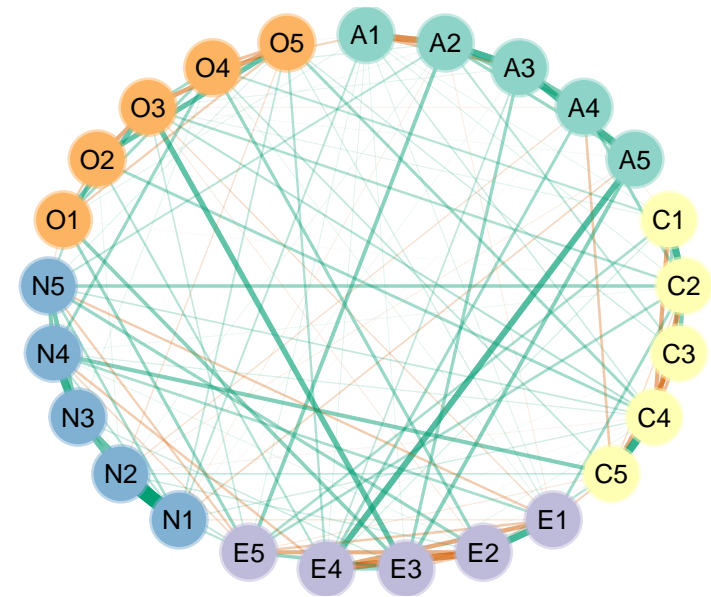

Local Linear Approximation

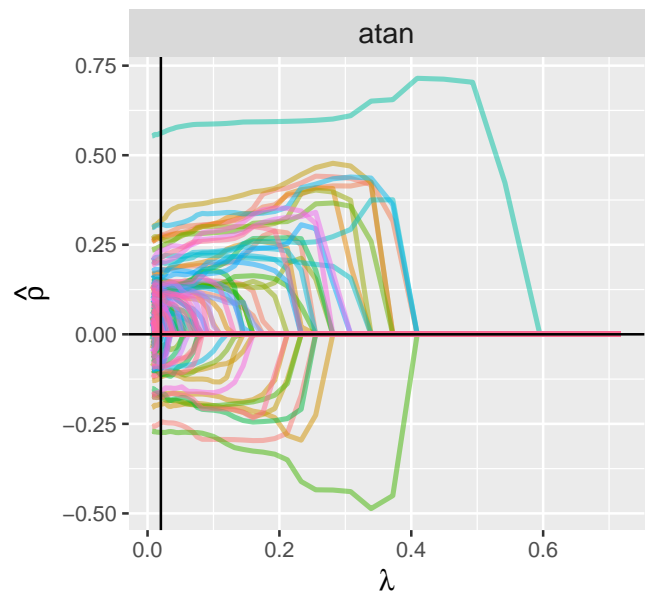

Edge Inclusion 'Probabilities'

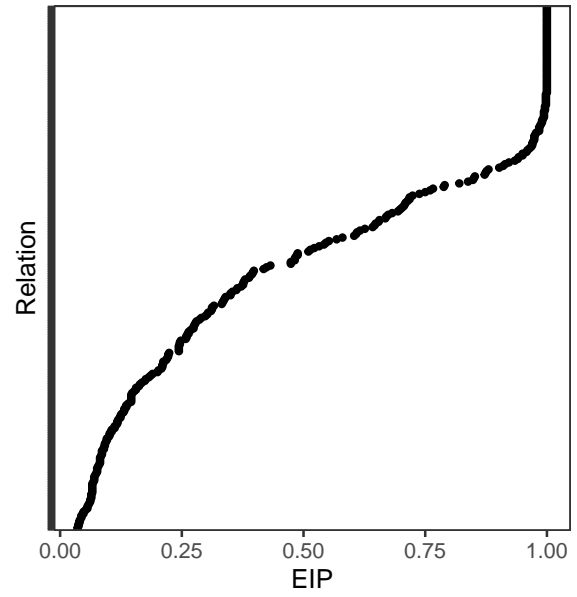

Figure 2: Illustrative plots.

terminated at that many steps or sooner if the convergence threshold is reached (defaults to thr = 1e-4). Note that a fully iterative LLA is not typically necessary (Fan et al., 2014).

Regularization Paths: The regularization paths can be visualized as follows:

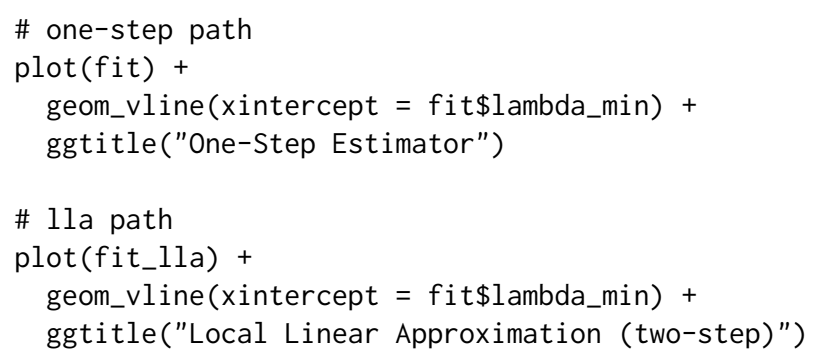

Figure 2 includes these regularization paths, where the partial correlations are plotted in relation to $\lambda$.

Conditional Dependence Structure: The estimated dependence structure is plotted as follows:

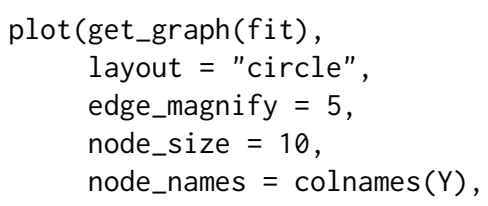




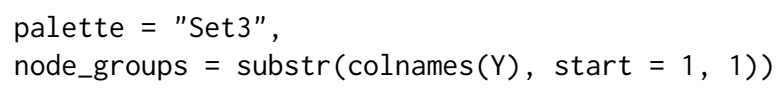

palette determines the color of the nodes according to node_groups () and edge_magnify () is used to increase the edge sizes. This plot is provided in Figure 2.

Edge Inclusion "Probabilities": $\quad$ As outlined in Hastie et al. (2015, see Figure 6.7 therein), a nonparametric bootstrap can be used to compute inclusion "probabilities." For each bootstrap sample, the basic idea is to select a graph and then tally the number of times each edge was selected, expressed as a proportion of the number of bootstrap samples:

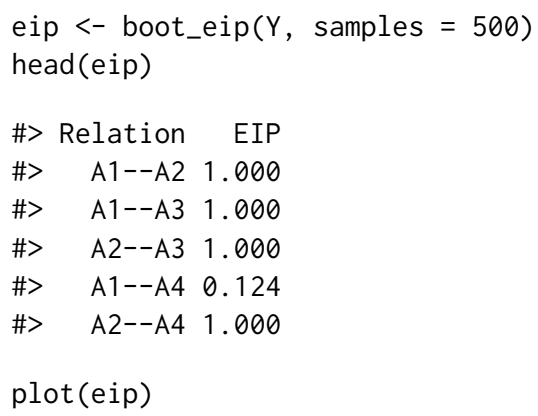

This plot is provided in Figure 2.

\section{False Discovery Control}

The debiased estimator, described above, is implemented with

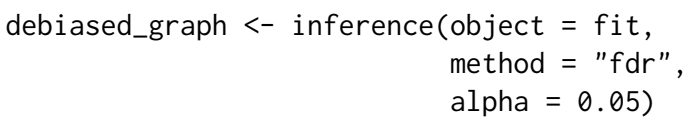

\# debiased precision matrix

debiased_graph\$Theta

\# adjacency matrix

debiased_graph $\$ a d j$

\# uncorrected pvalues

debiased_graph\$uncorrect

\# correct pvlaues

debiased_graph\$correct

Note that method is passed to $p$.adjust for correcting the $p$-values. Additionally, the dependence structure can be visualized with plot (get_graph(debiased_graph)).

\section{Confirmatory Testing}

GGMncv includes the function confirm_edges() that allows for testing specific edges that were first detected with data-driven model selection. In this way, the total number of tests is reduced which translates into more statistical power when confirming the edges in an independent dataset.

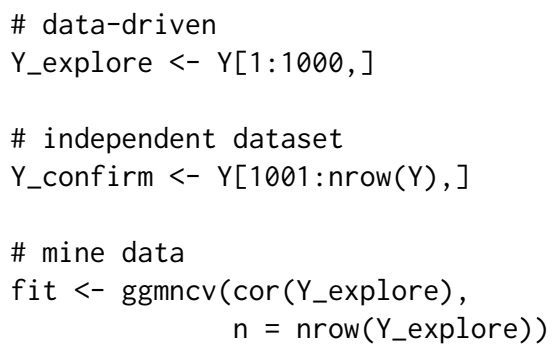




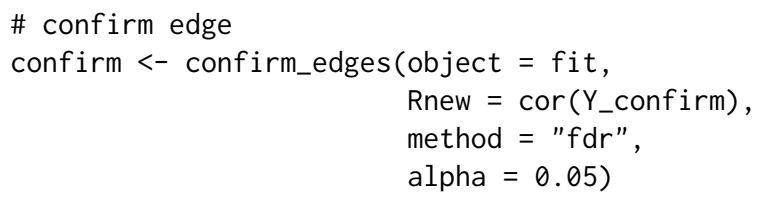

The object confirm is of class ggmncv which allows for using all the print and plotting functions. Note confirmatory testing is accomplished by fixing the regularization parameter (i.e., $\lambda=\sqrt{\log (p) n}$ ), thereby avoiding data-driven selection, and setting penalty = "lasso". This is the approach described in Janková and van de Geer (2015).

\section{Added Bonus: Penalized Least Squares}

There is direct correspondence between the elements of $\boldsymbol{\Theta}$ and multiple regression. First let each node $V_{j}$ be defined as $\mathbf{y}$-that is, the scores of the $n$ subjects on the $j$ th variable (node). Each node is regressed on the remaining $p-1$ variables, which estimates the "neighborhood" for each node

$$
\mathbf{y}=X \beta_{j}+\varepsilon,
$$

where $\varepsilon$ is an $n$-dimensional vector, with the mean as a vector of zeroes, and the covariance matrix as $\sigma^{2} \mathbf{I}_{n}$. Here $\boldsymbol{X}$ is a $n \times(p-1)$ design matrix, that excludes the $i$ th node, and $\boldsymbol{\beta}$ is $(p-1) \times 1$ vector. The intercept is excluded, due to standardizing the data, so $\boldsymbol{\beta}^{(j)}$ contains $p-1$ regression coefficients. To be clear, $\boldsymbol{\beta}_{j}$ denotes the vector of coefficients for the $j$ th regression model, where the individual elements are defined as $\beta_{i j}$. The residuals are assumed to follow $\mathcal{R} \sim \mathcal{N}\left(0, \sigma_{j}^{2}\right)$, where $\sigma_{j}^{2}$ is the residual variance for the $j$ th node. The regression coefficients and error variances then correspond to the off-diagonal and diagonal elements of $\boldsymbol{\Theta}$, that is,

$$
\theta_{i j}=\frac{-\beta_{i j}}{\sigma_{j}^{2}} \text { and } \theta_{j j}=\frac{1}{\sigma_{j}^{2}},
$$

where $\theta_{i j}$ denotes the covariance corresponding to $i$ th row and $j$ th column of $\boldsymbol{\Theta}$. Hence, for Gaussian data, GGMncv can be used for multiple regression for these nonconvex penalties. To my knowledge, this is the only implementation in $\mathrm{R}$ for those penalties that approximate the $\ell_{0}$-penalty. The regression coefficients are computed simply as coef(fit) and it is possible to evaluate, say, out-of-sample predictions, using predict().

\section{Summary}

This paper described the GGMncv package for estimating Gaussian graphical models with nonconvex regularization. In the future, I plan to extend the range of penalties as they become available.

\section{Bibliography}

H. Akaike. A New Look at the Statistical Model Identification. IEEE Transactions on Automatic Control, 19(6):716-723, 12 1974. ISSN 15582523. doi: 10.1109/TAC.1974.1100705. [p6]

K. Baba and M. Sibuya. Equivalence of Partial and Conditional Correlation Coefficients. J. Japan Statist. Soc., 35(1):1-19, 2005. [p2]

K. Baba, R. Shibata, and M. Sibuya. Partial Correlation and Conditional Correlation as Measures of Conditional Independence. Australian \& New Zealand Journal of Statistics, 46(4):657-664, 2004. ISSN 1369-1473. doi: 10.1111/j.1467-842X.2004.00360.x. [p2]

R. Berk, L. Brown, A. Buja, K. Zhang, and L. Zhao. Valid post-selection inference. Annals of Statistics, 41(2):802-837, 2013. ISSN 00905364. doi: 10.1214/12-AOS1077. [p7]

D. Borsboom, A. O. J. Cramer, V. D. Schmittmann, S. Epskamp, and L. J. Waldorp. The Small World of Psychopathology. PLoS ONE, 6(11):e27407, 2011. ISSN 1932-6203. doi: 10.1371/journal.pone.0027407. [p1]

P. Breheny. The group exponential lasso for bi-level variable selection. Biometrics, 71(3):731-740, 2015. [p4] 
P. Breheny and J. Huang. Coordinate descent algorithms for nonconvex penalized regression, with applications to biological feature selection. Annals of Applied Statistics, 5(1):232-253, 2011. ISSN 19326157. doi: 10.1214/10-AOAS388. [p1,2,3]

L. Breiman. Heuristics of instability and stabilization in model selection. The annals of statistics, 24(6): 2350-2383, 1996. [p4]

J. Chen and Z. Chen. Extended Bayesian information criteria for model selection with large model spaces. Biometrika, 95(3):759-771, 2008. ISSN 0006-3444. doi: 10.1093/biomet/asn034. [p6]

G. Costantini, S. Epskamp, D. Borsboom, M. Perugini, R. Mõttus, L. J. Waldorp, and A. O. Cramer. State of the aRt personality research: A tutorial on network analysis of personality data in R. Journal of Research in Personality, 54:13-29, 2015. ISSN 10957251. doi: 10.1016/j.jrp.2014.07.003. [p7]

A. Dempster. Covariance Selection. Biometrics, 28(1):157-175, 3 1972. ISSN 0006341X. doi: 10.2307/ 2528966. [p1]

L. Dicker, B. Huang, and X. Lin. Variable selection and estimation with the seamless-L0 penalty. Statistica Sinica, 23(2):929-962, 2013. ISSN 10170405. doi: 10.5705/ss.2011.074. [p3, 4]

M. Drton and M. D. Perlman. Multiple Testing and Error Control in Gaussian Graphical Model Selection. 22(3):430-449, 2005. ISSN 0883-4237. doi: 10.1214/088342307000000113. [p2]

J. Fan and R. Li. Variable Selection via Nonconcave Penalized Likelihood and its Oracle Properties. Journal of the American Statistical Association, 96(456):1348-1360, 2001. doi: 10.1198/016214501753382273. $[\mathrm{p} 2,3]$

J. Fan, Y. Feng, and Y. Wu. Network exploration via the adaptive LASSO and SCAD penalties. The annals of applied statistics, 3(2):521, 2009. [p2, 5, 6]

J. Fan, L. Xue, and H. Zou. Strong oracle optimality of folded concave penalized estimation. Annals of Statistics, 42(3):819-849, 2014. ISSN 00905364. doi: 10.1214/13-AOS1198. [p8]

J. Fan, Y. Liao, and H. Liu. An overview of the estimation of large covariance and precision matrices. Econometrics Journal, 19(1):C1-C32, 2016. ISSN 1368423X. doi: 10.1111/ectj.12061. [p1]

J. Fan, Y. Feng, and L. Xia. A projection-based conditional dependence measure with applications to high-dimensional undirected graphical models. Journal of Econometrics, 2020. [p1]

D. P. Foster and E. I. George. The risk inflation criterion for multiple regression. The Annals of Statistics, pages 1947-1975, 1994. [p6]

R. Foygel and M. Drton. Extended Bayesian Information Criteria for Gaussian Graphical Models. Advances in Neural Information Processing Systems, pages 604-612, 2010. [p6]

J. Friedman, T. Hastie, and R. Tibshirani. Sparse inverse covariance estimation with the graphical lasso. Biostatistics, 9(3):432-441, 2008. ISSN 14654644. doi: 10.1093/biostatistics/kxm045. [p1]

P. Giudici and A. Spelta. Graphical network models for international financial flows. Journal of Business $\mathcal{E}$ Economic Statistics, 34(1):128-138, 2016. [p1]

T. Hastie, R. Tibshirani, and M. Wainwright. Statistical Learning with Sparsity. Statistical Learning with Sparsity, 33(1), 2015. doi: 10.1201/b18401. [p9]

S. Højsgaard, D. Edwards, and S. Lauritzen. Graphical Models with R. 2012. ISBN 1461422981. doi: 10.1007/978-1-4614-2299-0. [p2]

J. Janková and S. van de Geer. Confidence intervals for high-dimensional inverse covariance estimation. Electronic Journal of Statistics, 9:1205-1229, 2015. ISSN 19357524. doi: 10.1214/15-EJS1031. [p7, 10]

A. Javanmard and A. Montanari. Confidence Intervals and Hypothesis Testing for High-Dimensional Regression. pages 1-21, 2013. ISSN 15337928. [p7]

A. Javanmard and A. Montanari. De-biasing the Lasso: Optimal Sample Size for Gaussian Designs. arXiv, 2015. [p7]

D. Kim, S. Lee, and S. Kwon. A unified algorithm for the non-convex penalized estimation: The ncpen package. arxiv, 2018. [p1]

Y. Kim, S. Kwon, and H. Choi. Consistent Model Selection Criteria on High Dimensions. Journal of Machine Learning Research, 13:1037-1057, 2012. ISSN 15324435. [p6] 
S. L. Lauritzen. Graphical models, volume 17. Clarendon Press, 1996. [p2]

O. Ledoit and M. Wolf. A well-conditioned estimator for large-dimensional covariance matrices. Journal of Multivariate Analysis, 88(2):365-411, 2004. ISSN 0047259X. doi: 10.1016/S0047-259X(03)00096-4. [p6]

J. Lv, Y. Fan, and others. A unified approach to model selection and sparse recovery using regularized least squares. The Annals of Statistics, 37(6A):3498-3528, 2009. [p4]

R. Mazumder, J. H. Friedman, and T. Hastie. SparseNet: Coordinate descent with nonconvex penalties. Journal of the American Statistical Association, 106(495):1125-1138, 2011. ISSN 01621459. doi: 10.1198/ jasa.2011.tm09738. [p1]

R. J. McNally. Can network analysis transform psychopathology?, 2016. ISSN 1873622X. [p1]

A. Mohammadi and E. C. Wit. Bayesian structure learning in sparse Gaussian graphical models. Bayesian Analysis, 10(1):109-138, 2015. ISSN 19316690. doi: 10.1214/14-BA889. [p1]

M. Nikolova. Local strong homogeneity of a regularized estimator. SIAM Journal on Applied Mathematics, 61(2):633-658, 2000. [p4]

A. Ortiz, J. Munilla, I. Álvarez-Illán, J. M. Górriz, and J. Ramírez. Exploratory graphical models of functional and structural connectivity patterns for Alzheimer's disease diagnosis. Frontiers in Computational Neuroscience, 9(November):1-18, 2015. ISSN 16625188. doi: 10.3389/fncom.2015.00132. [p1]

W. Revelle. psych: Procedures for Psychological, Psychometric, and Personality Research, 2019. URL https: //cran.r-project. org/package=psych. [p7]

G. Schwarz and others. Estimating the dimension of a model. The annals of statistics, 6(2):461-464, 1978. [p6]

M. A. Sustik, B. Calderhead, and J. Clavel. glassoFast: Fast Graphical LASSO, 2018. URL https: //cran.r-project.org/package=glassoFast. [p6]

J. Taylor and R. J. Tibshirani. Statistical learning and selective inference. Proceedings of the National Academy of Sciences, 112(25):7629-7634, 2015. [p7]

R. Tibshirani. Regression Selection and Shrinkage via the Lasso, 1996. ISSN 00359246. [p1]

S. Van De Geer, P. Bühlmann, Y. Ritov, and R. Dezeure. On asymptotically optimal confidence regions and tests for high-dimensional models. Annals of Statistics, 42(3):1166-1202, 2014. ISSN 00905364. doi: 10.1214/14-AOS1221. [p7]

D. N. VanDerwerken. Variable Selection and Parameter Estimation Using a Continuous and Differentiable Approximation to the L0 Penalty Function. 2011. [p4]

Y. Wang and L. Zhu. Variable Selection and Parameter Estimation with the Atan Regularization Method. Journal of Probability and Statistics, 2016:1-12, 2016. doi: 10.1155/2016/6495417. [p4]

Y. Wang, Q. Fan, and L. Zhu. Variable selection and estimation using a continuous approximation to the L0 penalty. Annals of the Institute of Statistical Mathematics, 70(1):191-214, 2018. [p4]

H. Wickham. ggplot2: Elegant Graphics for Data Analysis. Springer-Verlag New York, 2016. ISBN 978-3-319-24277-4. URL http: //ggplot2.org. [p4]

D. R. Williams. Beyond Lasso: A Survey of Nonconvex Regularization in Gaussian Graphical Models. PsyArXiv, 2020. [p1, 3, 4, 5]

D. R. Williams and J. Mulder. Bayesian hypothesis testing for Gaussian graphical models: Conditional independence and order constraints. Journal of Mathematical Psychology, 99:102441, 2020. [p2]

D. M. Witten, J. H. Friedman, and N. Simon. New insights and faster computations for the graphical lasso. Journal of Computational and Graphical Statistics, 20(4):892-900, 2011. ISSN 10618600. doi: 10.1198/jcgs.2011.11051a. [p1]

C. H. Zhang. Nearly unbiased variable selection under minimax concave penalty. Annals of Statistics, 38(2):894-942, 2010. ISSN 00905364. doi: 10.1214/09-AOS729. [p3]

H. Zou and R. Li. One-step sparse estimates in nonconcave penalized likelihood models. Annals of Statistics, 36(4):1509-1533, 2008. ISSN 00905364. doi: 10.1214/009053607000000802. [p5, 6] 
Donald. $R$ Williams

Department of Psychology

University of California, Davis

Davis, California 95616, United States of America

Email:drwwilliams@ucdavis.edu 